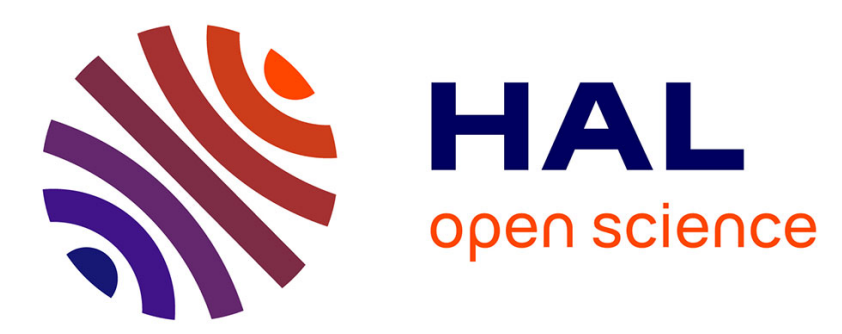

\title{
A Hybrid Concentric Tube Robot for Cholesteatoma Laser Surgery
}

Dang Viet Anh Nguyen, Cédric Girerd, Quentin Boyer, Patrick Rougeot, Olivier Lehmann, Laurent Tavernier, Jérôme Szewczyk, Kanty Rabenorosoa

\section{- To cite this version:}

Dang Viet Anh Nguyen, Cédric Girerd, Quentin Boyer, Patrick Rougeot, Olivier Lehmann, et al.. A Hybrid Concentric Tube Robot for Cholesteatoma Laser Surgery. IEEE Robotics and Automation Letters, 2021, 7 (1), pp.462 - 469. hal-03549460

\author{
HAL Id: hal-03549460 \\ https://hal.science/hal-03549460
}

Submitted on 31 Jan 2022

HAL is a multi-disciplinary open access archive for the deposit and dissemination of scientific research documents, whether they are published or not. The documents may come from teaching and research institutions in France or abroad, or from public or private research centers.
L'archive ouverte pluridisciplinaire $\mathbf{H A L}$, est destinée au dépôt et à la diffusion de documents scientifiques de niveau recherche, publiés ou non, émanant des établissements d'enseignement et de recherche français ou étrangers, des laboratoires publics ou privés. 


\title{
A Hybrid Concentric Tube Robot for Cholesteatoma Laser Surgery
}

\author{
D. V. A. Nguyen ${ }^{1}$, C. Girerd ${ }^{2}$, Q. Boyer ${ }^{1}$, P. Rougeot ${ }^{1}$, O. Lehmann ${ }^{1}$, L. Tavernier ${ }^{3}$, J. Szewczyk ${ }^{4}$, \\ and K. Rabenorosoa ${ }^{1}$
}

\begin{abstract}
This letter presents a hybrid concentric tube robot which covers the middle ear volume for exhaustive ablation of residual cholesteatoma. The proposed robotic system combines a concentric tube robot and a wrist at the distal end, actuated by a tendon. We first introduce the surgical protocol through two access points (ear canal and few millimeters size hole through the mastoid), then derive the anatomical constraints and specify the robot tasks. Based on the robot model enriched with the optical fiber stiffness and on anatomical constraints, the robot parameters are determined as the ones among discretized sets that provide the maximal volume coverage. Experiments are conducted with a benchtop prototype on a 3D printed middle ear phantom to validate the wrist model with the optical fiber and the robot repeatability assessment. The wrist model achieved an root mean square error (RMSE) of $1.33^{\circ}$ and $R^{2}=96.8 \%$. The robot repeatability has an RMSE of $0.7 \mathrm{~mm}$ for distance errors and $1.34^{\circ}-2.42^{\circ}-3.11^{\circ}$ for the angular ones. We finally demonstrated the ablation of cholesteatoma by the embedded optical fiber on the hybrid concentric tube robot prototype.
\end{abstract}

Index Terms-Medical robots and systems, steerable catheters/needles, mechanism design, middle ear surgery.

\section{INTRODUCTION}

C HOLESTEATOMA is a serious form of chronic otitis presented as an inflammatory pseudo-tumor characterized by proliferation of epidermal tissue, with desquamation and destruction of surrounding bones. It generally begins in the upper part of the tympanic membrane, expands into the middle ear, filling in the eardrum cavity around the ossicles. The first symptoms are usually continual ear drainage. Although rare, complications can be as important as dizziness, meningitis (or other brain infections), facial palsy or total and irreversible deafness [1], [2]. The affected anatomical area, namely the middle ear, is located in a confined space, and requires the use of miniature tools embedded on dexterous

Manuscript received: September 3, 2021; Accepted November 1, 2021.

This paper was recommended for publication by Editor Jessica BurgnerKahrs upon evaluation of the Associate Editor and Reviewers' comments. This work was supported by the regional BFC project CoErCIVe, the EIPHI Graduate School (contract "ANR-17-EURE-0002") and ANR $\mu$ RoCS (contract "ANR-17-CE19-0005"). We would like to thank Pierre Roux and Gerard Michel for technical support on prototype fabrication.

${ }^{1}$ Authors are with FEMTO-ST Institute, Univ. Bourgogne Franche-Comté, CNRS, Besançon, France. \{dang.nguyen, patrick.rougeot, olivier.lehmann, rkanty\}efemto-st.fr

2 Author is with the Department of Mechanical and Aerospace Engineering, University of California, San Diego, La Jolla, CA 92093 USA. cgirerdeeng.ucsd.edu

3 Author is with Univ. Hospital of Besançon, Univ. Bourgogne FrancheComté, France. Itavernier@chu-besancon.fr

4 Author is with Institute for Intelligent Systems and Robotics (ISIR), Sorbonne University, CNRS, France. szewczykeisir.upmc. fr

Digital Object Identifier (DOI): see top of this page. robotic systems for access and volume coverage. To date, this type of intervention has been achieved by an open surgery called a mastoidectomy [3], [4], using rigid surgical tools. However, the associated risk of recurrences due to residual cholesteatoma can reach $23-25 \%$ of cases depending on the country and age group [1], [5]. Follow-up strategy is systematically applied and induces a painful second examination of the patient, along with additional costs. The complete removal of cholesteatoma is thus a considerable limitation of current practices due to the limited miniaturization and dexterity of the surgical tools [1], [4].

Prior works suggest that minimally invasive surgery in the ear requires the use of robotic devices, with examples that include middle ear surgery [6]-[8], cochlear implant in the inner ear using a tubular manipulator [9], magnetic guidance [10], and sensor-based computer assisted surgery with recent tests in humans [11]. However, as previously mentioned, the confined surgical area causes difficulties in obtaining a complete removal of cholesteatoma, especially for hard-to-reach locations inside the middle ear. The aim of this work is thus to develop a dexterous continuum robot that can explore the middle ear and remove the residual cholesteatoma with laser ablation by using two accesses to the middle ear.

The robot has to achieve a large curvature at the tip, in order to be able to sweep the desired volume and enable contact with hard-to-reach anatomical targets. In the literature, notched tube compliant joints (also called wrist), were used to enhance the dexterity of the robot tip for endoscopy and minimally invasive surgical interventions. They are fabricated by cutting notches into a super-elastic tube, and are driven with an internal tendon [12]-[14]. With a diameter of 1 to $2 \mathrm{~mm}$, they are suitable for operation in confined spaces like the middle ear. However, to ensure a collision-free deployment as well as access to hard-to-reach locations for cholesteatoma removal, the robot needs to have more than a single curved segment. Chitalia et al. proposed a multi-segment notched tube manipulator for pediatric neuroendoscope [15]. The proposed design successfully improves the dexterity of the robot. However, the fixed relative angle (about the centerline axis) between the bending directions of the curved segments still limits the accessible zone of the robot tip. Instead, we propose to use concentric tube robots (CTRs) which constitute good candidates for highly constrained volumes, with previous work that demonstrated the feasibility of the inspection of the olfactory epithelium [16], insertion of cochlear implants [17], optic nerve sheath fenestration [18], among others. The independent rotations of the tubes allow the robot to have multiple bending 
directions in space. Another interesting feature of these robots is their ability to deploy in a follow-the-leader manner, where the body of the robot follows the path traced out by its tip [19]. However, the fixed tube curvature of CTRs poses limitations regarding the volume that can be swept by the tip, while keeping the rest of the CTR body in a constrained environment. To improve the robot dexterity at the tip and increase its coverage volume while keeping the rest of the robot stationary, the distal curvature of the robot thus needs to be varied. In order to achieve that, we propose a continuum robot with hybrid actuation. This concept combines a needlesize wrist with tendon driven actuation on the innermost tube of a CTR, originally presented in [12] for an endonasal surgery. The comparison of accessible spaces using different continuum robot designs is demonstrated in this letter.

The main contributions of this paper are 1) the proposition of a hybrid CTR for cholesteatoma laser surgery by considering two accesses to the middle ear in order to ensure a maximum coverage; 2) the formulation of design considerations, modeling, parameterization, and fabrication of the highly dexterous, cannula-like continuum robot integrated with a laser instrument to perform cholesteatoma ablation; 3) the experimental contributions include the validation of the wrist design in the presence of the embedded optical fiber (OF), the sensitivity assessment of the wrist to parameters dependent on machining tolerances, and the measurement of the robot repeatability; 4) the demonstration of cholesteatoma ablation with green laser on a benchtop prototype.

The paper is organized as follows: the anatomical constraints and robot tasks are presented in Section II. The modeling of the hybrid concentric tube robot with a wrist at the tip is established in Section III. Then, Section IV focuses on the selection of a robot design to ensure a maximum coverage of the volume of interest. In Section V, we present experimental tests to validate the robot design and illustrate the ablation of the cholesteatoma cells by LBO laser. Finally, the conclusion and perspectives of this work are presented in Section VI.

\section{AnATOMicAl CONSTRAints AND RoBot TASKS}

In this section, we present the area of interest, and detail the tasks that must be performed by the robot to navigate inside it and remove the residual cholesteatoma.

\section{A. Anatomical constraints}

As previously mentioned, the anatomical area of interest is the middle ear, more specifically the tympanic cavity. In the anatomical representation of the ear shown in Fig. 1a, one can observe that the tympanic cavity has a pocket-like shape separated from the auditory canal by the eardrum. To ensure pressure wave transmission for hearing, the latter is linked to the cochlea by ossicles. There is a total of three accesses to the middle ear: through the auditory canal (which requires a temporary displacement or an opening of the tympanic membrane), through the eustachian tube via the nasal cavity, and through the mastoid (via a mastoidectomy or at least a bone hole) [20]. Among them, the transmastoid access

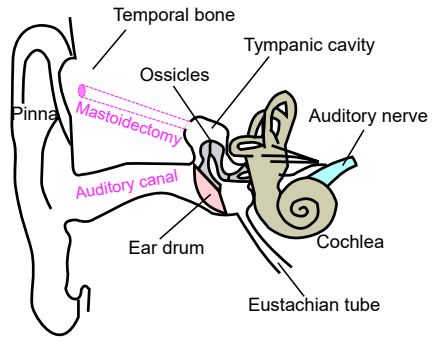

(a)

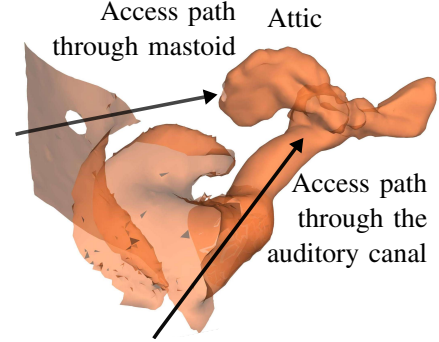

(b)
Fig. 1. (a) Anatomical representation of the ear, and (b) the meshed tympanic cavity that contains the cholesteatoma to remove with two accesses for the proposed surgical protocol.

to the attic (upper part of tympanic cavity) (Fig. 1b) is of major importance as it is the most frequent site of residual cholesteatoma [1]. To define the anatomical constraints, the model of the right ear shown in Fig. 1b was used. It is reconstructed from a set of DICOM images of the area of interest, taken with a CT scan. For this purpose, we used the image segmentation and 3D reconstruction software Invesalius [21]. Finally, the mesh was post-processed using MeshLab and SolidWorks to isolate, clean, and simplify it. The measured inner volume of the tympanic cavity of the obtained 3D model is $950 \mathrm{~mm}^{3}$, which is consistent with the typical values between 0.5 to $1 \mathrm{~cm}^{3}$ in the literature [22].

\section{B. Robot tasks}

The complete treatment in case of residual cholesteatoma requires two tasks. The first task consists in searching residual cholesteatoma by optical biopsy. We envision to use Optical Coherence Tomography (OCT) combined with fluorescence spectroscopy [23] to ensure compactness. Afterwards, the locations of the sites of interest would be sent to a machine learning classifier previously trained to determine whether or not the observed tissue is composed of residual cholesteatoma. The second task is to perform the ablation of the detected residual cholesteatoma by a green laser, demonstrated to be efficient [24]. The robot, which has to perform these two operations sequentially, is considered to be inserted through the mastoid (via a created hole of $3 \mathrm{~mm}$ in diameter) and through the auditory canal by displacing the eardrum. The ossicles are supposed to be dismantled beforehand, as the main challenge considered in this study is the middle ear coverage rather than path planning with obstacle avoidance to reach the desired site.

\section{MODELING OF THE HYBRID CONCENTRIC TUBE ROBOT}

The proposed robot consists of two main parts: the CTR, and the wrist actuated by a tendon. Both of these components are detailed in this section. We assume the deformation of the CTR to be negligible under the tendon load. This assumption holds for a CTR body that has a much higher stiffness than the wrist, which is the case in this work. Let $O$ be the point along the robot centerline that joins the wrist and the CTR (see Fig. 2). The homogeneous transformation matrix of the entire robot from the base to the tip is given by Eq. 1 .

$$
G_{B}^{T}=G_{B}^{O} G_{O}^{T}
$$




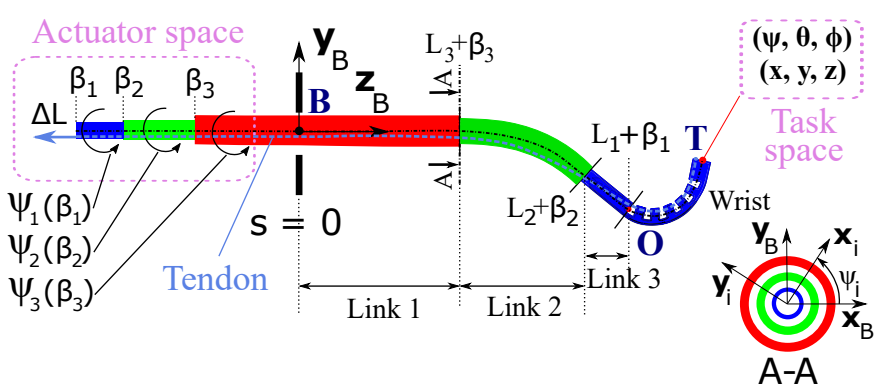

Fig. 2. Representation of the hybrid concentric tube robot composed of a CTR and a dexterous wrist integrated to the inner tube, with associated parameterization.

TABLE I

NOMENCLATURE

\begin{tabular}{ll}
\hline & Derivative with respect to $s$ \\
$N$ & Mapping from $\mathbb{R}^{3}$ to $\mathfrak{s o}(3)$ \\
$i$ & Number of tubes in the CTR \\
$s$ & Tube index, numbered in increasing diameter order \\
$k_{i b}$ & Curvilinear abscissa along the robot \\
$k_{i t}$ & Bending stiffness of tube $i$ \\
$L_{i}$ & Torsional stiffness of tube $i$ \\
$\beta_{i}$ & Total length of tube $i$ \\
$\boldsymbol{u}_{i}^{*}(s)$ & Location of the base of tube $i$ \\
$\boldsymbol{u}_{i}(s)$ & Initial prevurvature of tube $i$ \\
$\psi_{i}(s)$ & Equilibrium curvature of tube $i$ \\
$\boldsymbol{p}_{i}(s)$ & Twisting angle of tube $i$ \\
$R_{i}(s)$ & 3D position of tube $i$ in the global frame \\
$D_{2}$ & Orientation of tube $i$ in the global frame \\
$r_{i n}, r_{\text {out }}$ & Outer diameter of the tube 2 \\
$\rho$ & Inner and outer radii of the innermost tube \\
$l$ & Radius of the OF \\
$n$ & Length of the wrist \\
$h$ & Number of cutouts in the wrist \\
$c$ & Height of cut \\
$b$ & Distance between cuts \\
$g$ & Length of the distal uncut section \\
$d$ & Depth of cut \\
$\theta$ & Location of the OF centerlines within a cross-section \\
$\bar{y}$ & Bending angle within a cutout of the wrist \\
$\epsilon_{\text {max }}$ & Location of the neutral bending plane \\
$\mu_{s}$ & Maximum strain of the material \\
$\kappa, \kappa_{f}$ & Static friction coefficient between wrist and tendon \\
$E, E_{f}$ & Bending curvatures of the wrist and the OF \\
$F_{t e n d o n}$ & Young's modulus of the inner tube and the OF \\
\hline & Force applied on the tendon \\
\hline &
\end{tabular}

The main variables for the modeling are reported in Table I.

\section{A. Basics of CTR kinematics}

Torsionally-compliant kinematic models of CTRs have been derived from both energy minimization and Newtonian equilibrium of forces [25]. In the first case, the expression of the elastic energy stored inside the tube assembly leads to a boundary value problem (BVP). In this work, we consider tubes that have an initial precurvature vector of the form $\boldsymbol{u}_{i}^{*}(s)=\left[\begin{array}{lll}\kappa_{i} & 0 & 0\end{array}\right]^{T}$. The BVP to solve is given by Eq. (2) as introduced in [25]

$$
\left\{\begin{aligned}
\dot{\psi}_{i} & =u_{i z} \\
\dot{u}_{i z} & =\frac{k_{i b}}{k_{b} k_{i t}} \sum_{m=1}^{N} k_{m b} \kappa_{i} \kappa_{m} \sin \left(\psi_{i}(s)-\psi_{m}(s)\right),
\end{aligned}\right.
$$

with $k_{b}=\sum_{i=1}^{N} k_{i b}$. Continuity of the tube angles and their torsion must be ensured between each link, and the boundary conditions given by Eq. (3) apply at the insertion point $s=0$ of the tubes as well as at their tips, at $s=L_{i}+\beta_{i}$ [25].

$$
\left\{\begin{array}{l}
\psi_{i}(0)=\psi_{i}\left(\beta_{i}\right)-\beta_{i} \dot{\psi}_{i}(0) \\
\dot{\psi}_{i}\left(L_{i}+\beta_{i}\right)=0
\end{array}\right.
$$

The first boundary condition corresponds to the known tube angle at $s=0$, under the assumption of uniform torsion along the transmission lengths of the tubes, and knowing the angle at the tube base $\psi_{i}\left(\beta_{i}\right)$, which is a kinematic input. The second one corresponds to the absence of torsion at the free distal ends of the tubes. After solving for the torsion of the tubes along their lengths, their curvature components along the $x$ and $y$ axes are computed using Eq. (4).

$$
\boldsymbol{u}_{i x y}=\frac{1}{k_{b}} \sum_{m=1}^{N} R_{\psi_{m}-\psi_{i}} k_{m b} \boldsymbol{u}_{i x y}^{*}
$$

Then, after the final curvatures of the tubes $\boldsymbol{u}_{i}(s)$ are fully determined, the position of each tube in 3D space is obtained by integrating Eq. (5)

$$
\dot{\boldsymbol{p}}_{i}=R_{i} \boldsymbol{e}_{3}, \quad \dot{R}_{i}=R_{i} \hat{\boldsymbol{u}}_{i},
$$

where $\mathbf{e}_{3}$ is the vector of the Bishop frame tangent to the robot backbone, and $\hat{\boldsymbol{u}}_{i}$ is the skew-symmetric matrix computed from $\boldsymbol{u}_{i}(s)$, with initial conditions $\boldsymbol{p}(0)=\mathbf{0}$ and $R(0)=R_{z}\left(\psi_{i}(0)\right)$. Thus, the transformation matrix $G_{B}^{O}$ can be obtained using the knowledge of the inner tube pose at $O$.

\section{B. Bending wrist mechanism}

The mechanism of the asymmetric notch joints (wrist) was originally described in [12] with both kinematics and statics models. In this section, the static one that maps the tendon tension, $F_{\text {tendon }}$, to the resulting bending angle, $\theta_{\text {total }}$, is briefly reviewed here for completeness, and then improved by taking into account the presence of the $\mathrm{OF}$ inside the wrist.

By using Castigliano's first theorem and considering the frictional loss of the tendon tension due to $2 n$ corners (two corners per cutout), the governing equation is given in Eq. 6

$$
F_{\text {tendon }}=\frac{1}{\eta^{2 n} L} \frac{\partial U(\kappa)}{\partial \theta_{\text {total }}},
$$

where $L$ is the moment arm length, $U(\kappa)$ denotes the total strain energy stored in the wrist which can be found by considering the piecewise linear stress-strain curve behavior of Nitinol as detailed in [12]. The tension lost due to friction at a corner $\eta$ can be expressed in terms of the static friction coefficient $\mu_{s}$ between the tendon and the wirst, and the deflection angle of the tendon $\gamma=\pi-\frac{\theta}{2}$ (Fig. 3c) using the static balance equation for a single corner, as in Eq. (7).

$$
\eta=\frac{\sin (\gamma / 2)-\mu_{s} \cos (\gamma / 2)}{\sin (\gamma / 2)+\mu_{s} \cos (\gamma / 2)}
$$


The relationship between $\theta_{\text {total }}$ and $\kappa$ is shown in Eq. 8 .

$$
\theta_{\text {total }}=n \theta=\frac{n h \kappa}{1+\bar{y} \kappa}
$$

in which the number of cutouts $n$, the cut height $h$ and the neutral bending plane location $\bar{y}$ are constant (see Fig. 3). The mapping between $F_{\text {tendon }}$ and $\theta_{\text {total }}$ can be obtained by numerically solving Eq. 6. The knowledge of $\theta$ (using Eq. 8) allows to calculate the homogeneous transformation $G_{O}^{T}$ from the connection point $O$ to the tip $T$ (Fig. 2).

\section{Optical fiber integration}

As the stiffness of the embedded OF cannot be neglected, the modeling of the wrist needs to be modified. Then, the strain energy of this fiber $U_{f}$ will be added in the total strain energy $U$ in Eq. 6. In the following, $U_{f}$ will be expressed as a function of $\theta_{\text {total }}$. For the purpose of similarity, the OF section contained in the wrist is assumed to be separated into two portions: straight $\left(S_{s}\right)$ and curved $\left(S_{c}\right)$ as shown in Fig. 4a. Then, the strain energy to be determined is stored in the curved portion $S_{c}$. Based on the constant curvature assumption, strain along the length of the OF varies in a cross section of the bending portion according to Eq. 9 .

$$
\epsilon_{f}=\kappa_{f}(y-d)=\kappa_{f} y_{f}
$$

The y-axis of the body coordinate frame lying at the centerline of the OF $y_{f}=y-d$ is employed now to simplify the expression (as shown in Fig. 4c). Considering a linear elastic deformation, the stress of the fiber can be found as

$$
\sigma_{f}\left(\epsilon_{f}\right)=E_{f} \epsilon_{f},
$$

where $E_{f}$ is the Young's modulus of the OF. By considering the curved portion of the OF as a set of one-dimensional stretched and compressed axial fiber elements, the strain energy density is given by Eq. 11 .

$$
W_{f}\left(\epsilon_{f}\right)=\int_{0}^{\epsilon_{f}} \sigma_{f}(e) d e=\frac{1}{2} E_{f} \epsilon_{f}^{2}
$$

An axial fiber element locating at $y_{f}$ has a length given by Eq. 12.

$$
l_{f}=\left(\kappa_{f}^{-1}+y_{f}\right) \theta_{\text {total }}
$$

The strain energy stored in the portion $S_{c}$ is given by Eq. 13 .

$$
U_{f}=\int_{-\rho}^{+\rho}\left(2 W_{f} l_{f} \sqrt{\rho^{2}-y_{f}^{2}}\right) d y_{f}=\frac{\pi E_{f} \kappa_{f} \rho^{4} \theta_{\text {total }}}{8}
$$

where $\rho$ is the radius of the OF. To obtain the relationship between $\kappa_{f}$ and $\kappa$, the length of the curved portion $S_{c}$ is approximated by Eq. 14 .

$$
S_{c}=\frac{\theta_{\text {total }}}{\kappa_{f}} \approx \frac{n h(1+\kappa d)}{1+\kappa \bar{y}}+(n-1) c
$$

By using Eq. 8, Eq. 13, and Eq. 14, the strain energy $U_{f}$ can be found as a function of $\theta_{\text {total }}$ according to Eq. 15 .

$$
U_{f}=\frac{\pi E_{f} \rho^{4} \theta_{\text {total }}^{2}}{8\left[n h+(n-1) c-\theta_{\text {total }}(\bar{y}-d)\right]}
$$

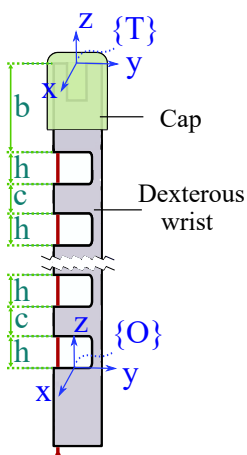

(a)

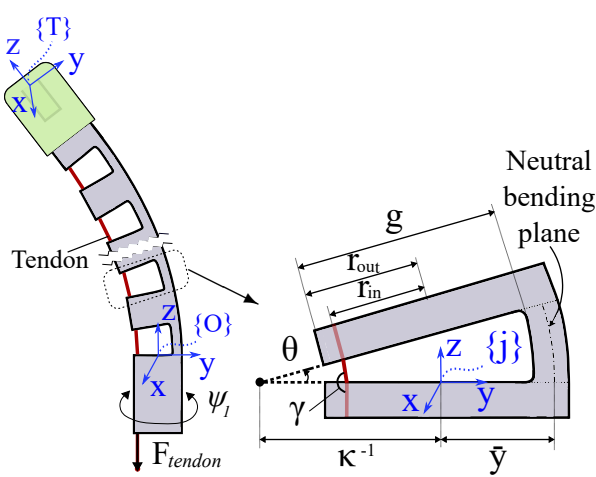

(b)

(c)
Fig. 3. Geometric representation of the wrist with its parameters and frames used for the model, with (a) the initial state of the wrist integrated at the tip of the innermost tube, (b) the wrist bends under the actuation force, and (c) a cutout of the bent wrist.

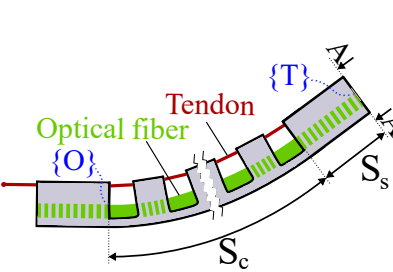

(a)

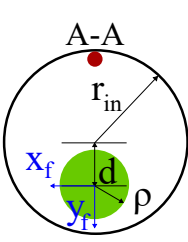

(b)

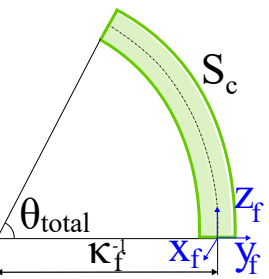

(c)
Fig. 4. (a) Bending wrist with the integrated OF, (b) cross-section of the wrist, and (c) curved portion of the OF.

\section{Robot SyNTHESIS BASED ON THE ANATOMY}

The main focus of this section is the selection of a robot that can cover the desired workspace inside the middle ear. For this, the volume to cover is compared to sets of sampled tip positions of the robot, corresponding to possible configurations. A configuration is called possible when it allows to reach a point inside the tympanic cavity without any collision with the anatomy (Fig. 5). The V-Collide library [26] is employed in this work to detect the collision between two volumes defined by triangular meshes. We also use the Parallel Computing Toolbox of Matlab (The MathWorks Inc., Natick, USA) to enhance the computation speed. The first volume is the mesh of the robot created with Matlab whose centerline is obtained using the model developed in Section III, and the second one is the area of interest of the ear as detailed in Section II.

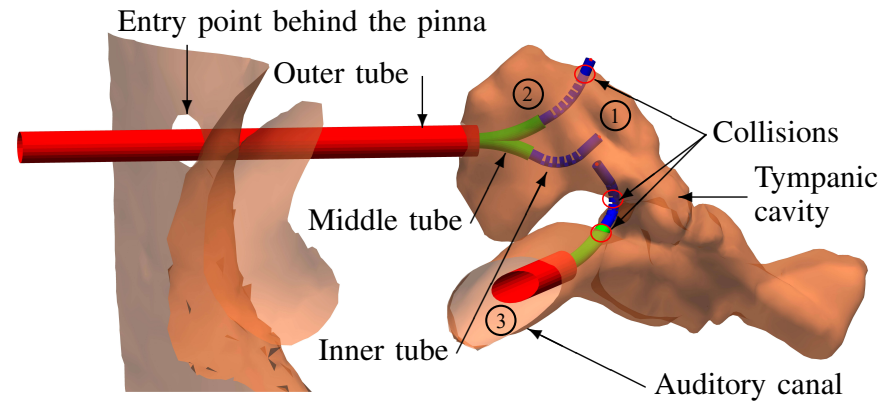

Fig. 5. Representation of robot configurations that reach the tympanic cavity through the two accesses. Configuration (1) is possible. Configuration (2) is not possible as the tip is outside the tympanic cavity. Configuration (3) is not possible because of collisions between the robot body and the ear model. 
TABLE II

CONFIGURATION OF THE CONCENTRIC TUBE ROBOT

\begin{tabular}{|c|c|c|c|c|c|c|}
\hline Tube & \multicolumn{2}{|c|}{ Outermost } & \multicolumn{2}{|c|}{ Middle } & \multicolumn{2}{|c|}{ Innermost } \\
\hline \multirow{2}{*}{$\begin{array}{l}\text { Diameter } \\
(\mathrm{mm})\end{array}$} & Outer & Inner & Outer & Inner & Outer & Inner \\
\hline & 2.50 & 1.80 & 1.50 & 1.25 & 1.08 & 1.00 \\
\hline \multirow{2}{*}{$\begin{array}{l}\text { Active } \\
\text { length } \\
(\mathrm{mm})\end{array}$} & \multicolumn{2}{|c|}{ Straight } & Straight & Curve & \multicolumn{2}{|c|}{ Straight } \\
\hline & \multicolumn{2}{|c|}{56} & $36-48$ & 20 & \multicolumn{2}{|c|}{$56-76$} \\
\hline \multirow{2}{*}{$\begin{array}{l}\text { Moving } \\
\text { ability } \\
\text { (deg-mm) }\end{array}$} & Rotary & Linear & Rotary & Linear & Rotary & Linear \\
\hline & \multicolumn{2}{|c|}{ fixed at the base } & full & 12 & full & 20 \\
\hline
\end{tabular}

The robot design parameters are chosen by selecting the set that increases the volume covered inside the ear. To do this, we create different configurations by changing discretely the translation and rotation of the tubes as well as the bending angle of the wrist. The reachable volume is then computed as the envelop of all tip points reached by the robot.

\section{A. Calculation of the coverage volume}

The collision test is performed in 3 steps. Firstly, the robot needs to be inserted through one of the entry paths of the tympanic cavity. Then, $\theta$ (see Fig. 3) is fixed to 0. The tubes are translated and rotated to obtain different robot configurations, and collision assessments with the ear are performed. The third step consists in collision tests between the mesh surface of the wrist by increasing $\theta$ with a discretized value from the non-colliding configurations at the previous step and the middle ear. As the number of mesh variables (vertices and faces) of the wrist is much smaller than those of the whole robot, the computation time is significantly reduced. Finally, the point cloud of the tip positions corresponding to possible solutions is saved. Its volume is determined using the alphaShape function in Matlab.

\section{B. Robot specifications}

In this section, we consider a CTR made of 3 tubes. It is a common choice for this type of robot, in order to obtain 6 degrees of freedom. As the mastoid bore and the auditory canal are both (nearly) straight, the outermost tube needs to be straight and substantially stiffer than the inner ones to constrain the robot to pass through these accesses. This requirement is considered valid if the proximal link has a stiffness 10 times higher than the adjacent one [27]. Next, the middle tube with a precurved shape is employed to navigate the robot inside the narrow space of the middle ear. Lastly, the innermost tube is chosen to be straight for the following reasons: 1) the combination of straight, curved and straight tubes ensures the absence of instabilities during the operation [28] which, if they occur, can be harmful to the patient; 2) only the wrist changes its shape when the innermost tube is rotated, which is a convenient way to decouple the motions of the tip compared to the rest of the robot body.

The tube diameters are reported in Table II according to the diameters of the entry hole through the mastoid, the tendon and the OF. The table also shows the requirements of the active length $(s>0)$ and the movement ability of the tubes. Stainless steel (Young's modulus E $=200 \mathrm{GPa}$ ) is selected for the outer tube to reduce its deflection due to the curvature of the middle tube. The material selected for the middle and inner tubes is Nitinol (E = $60 \mathrm{GPa})$ [29]. The design parameters of the CTR are the precurvature of the middle tube, $\kappa_{2}$. The maximum value of this quantity is $0.1 \mathrm{~mm}^{-1}$, given by Eq. (16) [30].

$$
\kappa_{2_{\max }}=\frac{2 \epsilon_{\max }}{D_{2}\left(1+\epsilon_{\max }\right)},
$$

where $D_{2}=1.5 \mathrm{~mm}$ is the outer diameter of the middle tube and $\epsilon_{\max }=8 \%$ is the recoverable strain limit of Nitinol.

For the wrist, the depth of cut $g$ is $0.93 \mathrm{~mm}$ to obtain a small strain on the tendon $(<4 \%)$ but still ensure low fatigue of the wrist material. The length of the distal uncut portion $b$ is chosen of $2 \mathrm{~mm}$. The height of cut and other uncut portions (named as $h$ and $c$ respectively (see Fig. 3a)) are designed with the same dimensions. It is important to note that if the height of cut $h$ is too large, the assumption of constant curvature will no longer hold, the risk of buckling-like failure will increase, and the friction loss will be greater [12]. Otherwise, if $h$ is too small, it will lead to a very sophisticated fabrication of the wrist and will limit its achievable curvatures. Thus, the value of $h$ is considered between 0.25 and $0.50 \mathrm{~mm}$. As the length

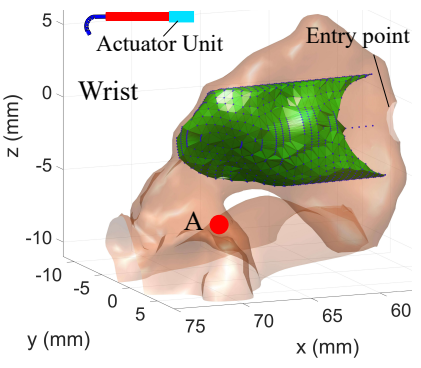

(a)

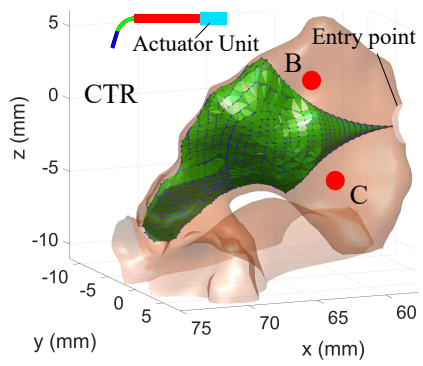

(b)

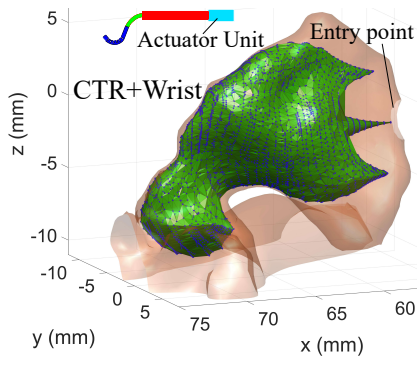

(c)

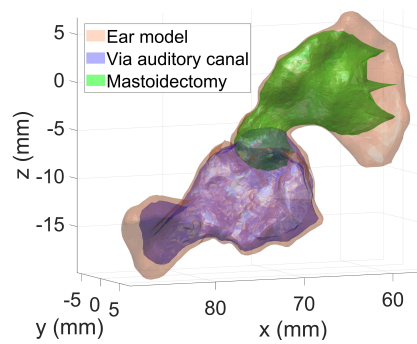

(d)

Fig. 6. Simulations of the tympanic cavity coverage volume by the robots that are inserted via a 3-mm hole through mastoid: (a) only 1-segment unidirectionally notched tube is considered, point $\mathrm{A}$ cannot be accessible by neither the robot that has only 1 wrist nor one that has 2 wrists in terms of $\mathrm{C}$ and $\mathrm{S}$ shapes as the inserting path requires bending in two different planes; (b) only the CTR is considered, the robot cannot reach point B and C due to the limitation of the precurvature of the tubes; (c) the CTR with the distal wrist significantly improves the coverage volume inside the tympanic cavity. (d) Total tympanic cavity coverage volume by the hybrid concentric tube robot inserted via the two accesses. 


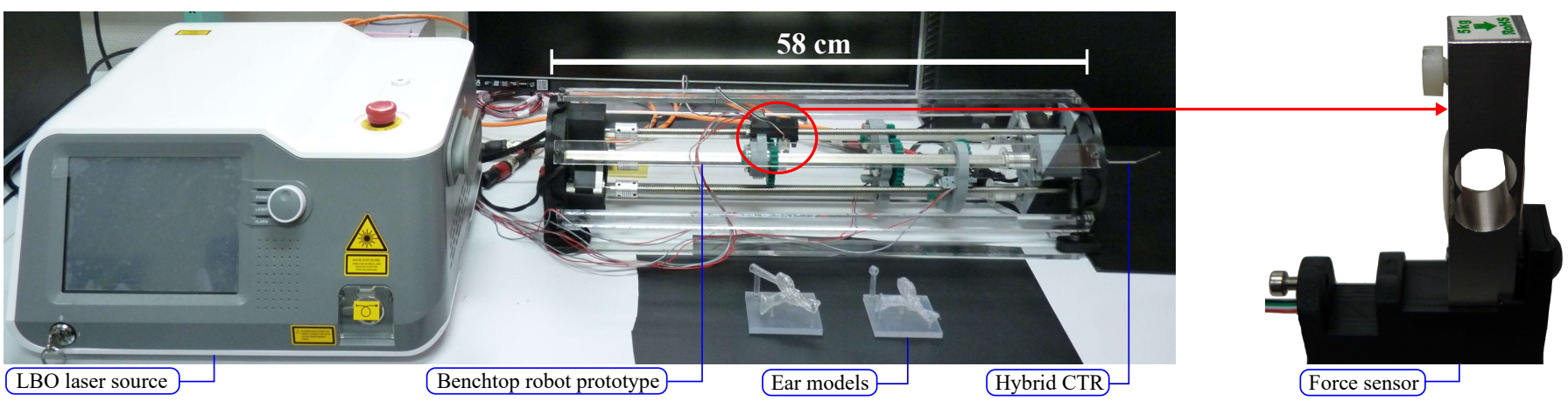

Fig. 7. Our experimental setup. The actuator unit consists of three part. Each part can perform a translation and a rotation. The outermost tube is fixed to the actuator unit's frame. Two linear-rotary stages are used to translate and rotate the middle and the innermost tubes. The last linear-rotary stage equipped with a force sensor is employed to drive the tendon and measure its tension force. Thus, 5 degrees of freedom is used in this letter.

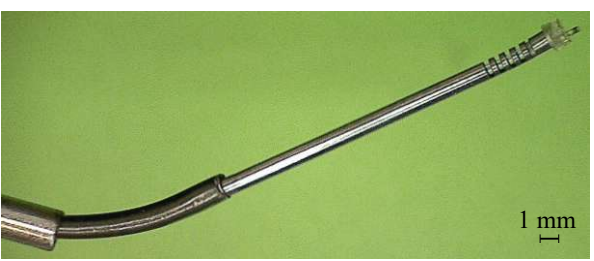

(a)

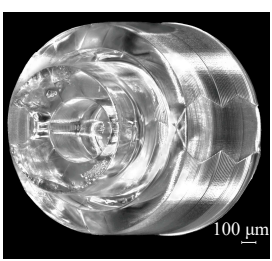

(b)
Fig. 8. (a) Hybrid CTR is built according to the simulation results. (b) The end cap to fix the tendon and the laser OF at the distal end.

of the upper part of the tympanic cavity is around $13 \mathrm{~mm}$, the wrist length $l$ (including the distal uncut portion) will be examined from 5 to $8 \mathrm{~mm}$ to find the best design which maximizes the coverage volume by inserting the robot through the entry hole on the mastoid. The number of cutouts $n$, which is now a variable depending on $l, h$, and $c$, will vary to obtain different values of $h$ in the mentioned survey interval. Based on the simulation result, $\kappa_{2}=0.1 \mathrm{~mm}^{-1}, l=5 \mathrm{~mm}$, and $n=5$ are chosen to fabricate the hybrid concentric tube robot for the purpose of the biggest coverage volume. The coverage volume obtained is $434 \mathrm{~mm}^{3}$ through the entry hole on the mastoid and $527 \mathrm{~mm}^{3}$ through the auditory canal. The total coverage volume is $884 \mathrm{~mm}^{3}$, which corresponds to $93.1 \%$ of the tympanic cavity volume (see Fig. 6d). In addition, Fig. 6 shows the comparison of accessible spaces using different robot designs through the created mastoid hole. The coverage volume using the hybrid CTR (434 $\mathrm{mm}^{3}$ (see Fig. 6c)) is almost twice as large as that obtained by only employing the wrist $\left(214 \mathrm{~mm}^{3}\right.$ (see Fig. 6a)) or the CTR $\left(220 \mathrm{~mm}^{3}\right.$ (see Fig. 6b)). One can confirm that hard-to-reach locations of the middle ear can be accessed by the hybrid CTR.

\section{EXPERIMENTAL ASSESSMENTS}

\section{A. Hybrid concentric tube robot prototype}

Experimental assessments were performed on a benchtop robot prototype (Fig. 7) which has already used as our validation robot for the kinematic model proposed in [31]. As a reminder, it is equipped with an actuation unit with 3 linear and 3 rotary stages using Beckhoff step motors with a positioning accuracy lower than $1 \mu \mathrm{m}$ and $0.02^{\circ}$ for the translation and rotation, respectively. The actuator unit is controlled through a program developed using Qt on a realtime GNU/Linux operating system (Xenomai) and an EtherCAT slave card. The tubes which constitute the CTR were acquired in a straight shape from Euroflex $\mathrm{GmbH}$. The shape-setting of the middle tube was performed using a mold and the tube specimen was placed in an air furnace at $600^{\circ} \mathrm{C}$ for $20 \mathrm{~min}$, and then quenched in water at room temperature. To fabricate the wrist, we used the Charmilles 2050 TW machine for wire-cut electrical discharge machining, with the diameter of the wire being $0.1 \mathrm{~mm}$. The hybrid CTR with design parameters selected according to the simulation results is shown in Fig. 8a. The cap of the wrist (diameter $=1.5 \mathrm{~mm}$ ) fabricated by utilizing Nanoscribe 3D printing has a specific design to constrain its relative rotation with respect to the wrist, the tip location of the tendon and also the optical fiber (Fig. 8b). We use an LBO laser generator from L.I.S. company (France) with the power up to $8 \mathrm{~W}$ and the wavelength of $532 \mathrm{~nm}$ for cholesteatoma ablation (see Fig. 7).

\section{B. Model validation \& Sensitivity analysis of the wrist}

An experiment is conducted to validate the model of the wrist. The M-112 compact micro-translation stage from Physik Instrumente (Germany) with $0.05 \mu \mathrm{m}$ resolution was used to move the tendon and a force sensor (Phidgets CZL635) with $25 \mathrm{mN}$ resolution measured the force applied on the tendon. We used a digital camera (DNT DigiMicro 2.0) to captured the images of the bending wrist. The experimental comparison with the statics model is shown in Fig. 9a. To illustrate the sensitivity of the model to the cut depth $g$ and the static friction coefficient $\mu_{s}$ which mainly depend on the machining tolerances, we fabricated 3 different wrists with the same nominal design parameters as mentioned in Section IV and determined these two parameters that provided the best agreement with the experimental results. As shown in Fig. 9, a small variation in these parameters (within 1\%) can lead to a non negligible difference in the bending of the wrists. One can conclude that the tolerance of the machining method is really important and a calibration step is mandatory before the use of the wrist. Now, we use wrist 2 for the following experiments. Fig. 10a shows the validation tests of the tip spatial trajectory with $R M S E=0.708 \mathrm{~mm}$. To fit the wrist model to the 


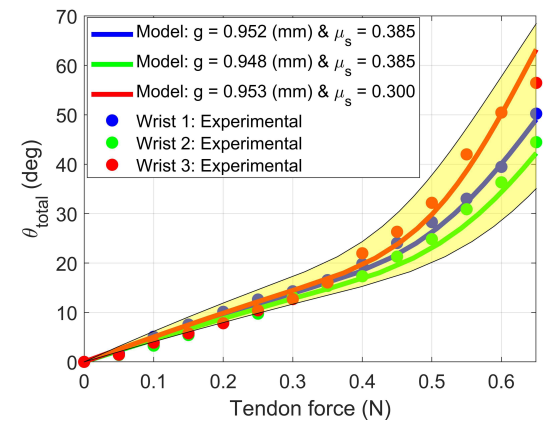

(a)

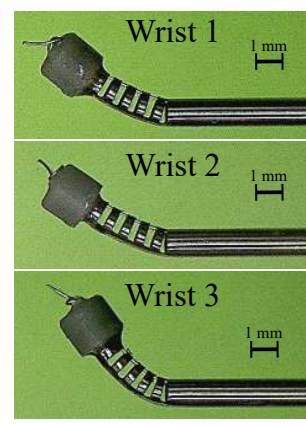

(b)
Fig. 9. (a) The statics model predicted and experimental results correspond to the tendon force required to bend the wrists. We conducted three tests for each wrists by changing the speed of the translation stage to 5,10 , and $15 \mu \mathrm{m} / \mathrm{s}$ and calculate the average value for the experimental data. The yellow domain shows the sensitivity of the model in blue curve with $1 \%$ variation of $g$ and $\mu_{s}$. As the statics model is most sensitive to the cut depth, the red curve (wrist 3) with a big difference in $\mu_{s}$ is still belong to the yellow domain. (b) Wrist 1,2 and 3 under the tendon force of $0.6 \mathrm{~N}$.

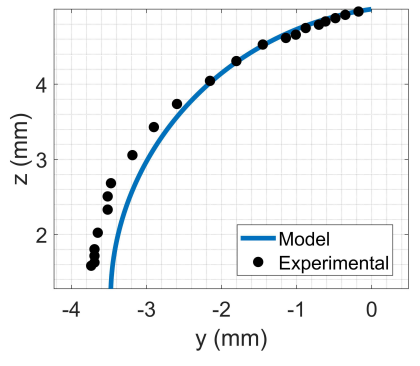

(a)

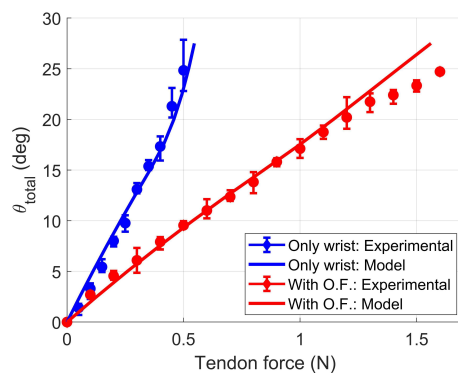

(b)
Fig. 10. (a) Model predicted and experimental tip spatial trajectory of the wrist (without the OF for a maximum bending of $85^{\circ}$ ). The error arises when the wrist bends with a big curvature as the proximal cutout firstly bends during the test. (b) Model predicted and experimental results correspond to the tendon force in case of with and without the OF. We increase the bending angle of the wrist until the maximum value is obtained $\left(27^{\circ}\right.$ for wrist with OF). Parameters of the model: $\rho=0.42 \mathrm{~mm}, d=0.12 \mathrm{~mm}$, and $L=R_{i}$.

experimental results, we estimate the Young's modulus of the OF $E_{f}=3.1 \mathrm{GPa}$. This estimated value is consistent with one reported in the literature (about $3 \mathrm{GPa}$ ) [32]. The static model is validated with $R M S E=1.478^{\circ}\left(R^{2}=98.7 \%\right)$ and $R M S E=1.330^{\circ}\left(R^{2}=96.8 \%\right)$ in case of without the embedded OF and with it, respectively (Fig. 10b). The tendon force significantly increases in the presence of the $\mathrm{OF}$ that shows the importance of considering this fiber in the model. The coverage volume obtained in Section IV is an ideal case. As shown in the experiment, the maximum curvature of the wrist is restricted by the allowable bending curvature the $\mathrm{OF}$, thus a small bending radius must be an essential requirement of the OF for the future compact design (see Section VI).

\section{Robot repeatability}

According to the surgical protocol, the proposed robot has to perform sequentially the cholesteatoma detection and removal. Thus, the ability to return to the same position between these two phases is an indispensable requirement of the robot. In this section, we propose an efficient method to

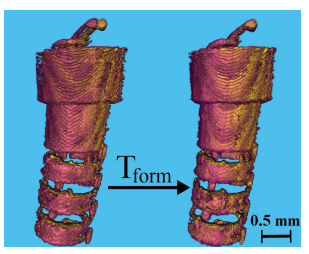

(a)

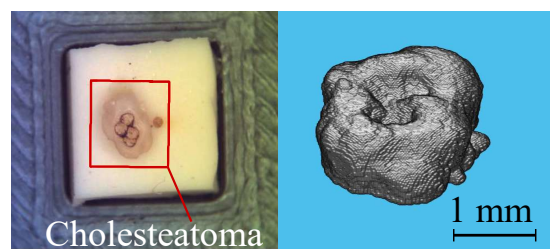

(b)
Fig. 11. OCT volume images (C-scan). (a) Left: Two different OCT images of the same actuation set. Right: After applying the transformation matrix for one image to align it with the other one. (b) Left: cholesteatoma cells burned by using the LBO laser. Right: volume image of the residual cholesteatoma during the ablation.
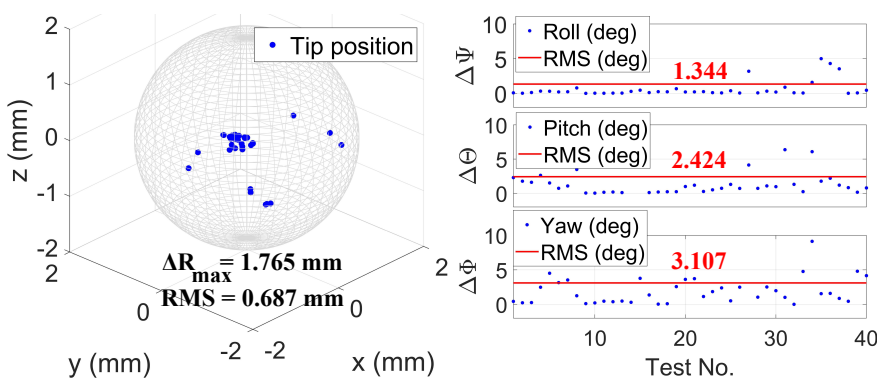

Fig. 12. The measurement of repeatability value of the robot.

measure the repeatability of the hybrid CTR without adding any external sensor which can cause unexpected errors in the robot configuration at millimeter scale. This method is based on using OCT volume image (C-scan) of the distal part of the robot and perform the point cloud registration as previously presented in [33]. A set of 40 volume images was obtained with a Telesto-II 1325nm spectral domain from ThorLabs by translating, rotating the tubes as well as bending the wrist, then returning to the same programmed configuration. The purpose of this method is to find the transformation matrix $\mathrm{T}_{\text {form }} \in \mathrm{SE}(3)$ to align the moving point cloud (volume image) with the reference point cloud. The first volume image was employed as the reference and the function imregtform of MATLAB was used to estimate the geometric transformation matrix as shown in Fig. 11a. Fig. 12 shows the experimental result where RMSE is $0.7 \mathrm{~mm}$ for distance errors and $1.34^{\circ}$ $2.42^{\circ}-3.11^{\circ}$ for the angular ones. These obtained results are acceptable for the requirements of the cholesteatoma ablation in a semi-automated mode.

\section{Cholesteatoma removal demonstration}

To demonstrate the ability of the proposed robot to fulfill the surgical intervention, real cholesteatoma cells collected after surgical procedures from Besançon Hospital were used in this experiment. Through teleoperated mode, the proposed robot is moved to reach the target location and perform the ablation of the cholesteatoma by LBO laser. The movement sequence and the ablation are available in the accompanying media. In addition, we can monitor the volume of the residual cholesteatoma cells by using the OCT C-scan and image processing methods [33]. An example of result is shown in Fig. $11 \mathrm{~b}$ where the estimated volume is about $2.399 \mathrm{~mm}^{3}$. 


\section{CONCLUSION}

In this letter, a hybrid concentric tube robot with a tendon driven wrist is proposed for cholesteatoma laser surgery. The proposed surgical protocol was based on two accesses: a $3 \mathrm{~mm}$ hole through the mastoid and the auditory canal. A complete modeling of the robot with the embedded laser surgical tool has been derived. Then, the robot design integrated the model of the hybrid CTR, discretization of the design parameters, and collision test during deployment in the middle ear. Simulation results demonstrated that the hybrid CTR was an efficient design to contact hard-to-reach locations inside the tympanic cavity. The dexterous wrist model was validated in the present of the optical fiber with $R M S E$ about $1.33^{\circ}$. The robot repeatability was assessed using OCT volume image where RMSE is $0.7 \mathrm{~mm}$ for distance errors and $1.34^{\circ}-2.42^{\circ}-3.11^{\circ}$ for the angular ones. The ablation of real cholesteatoma cells by using LBO laser was demonstrated. Future work will focus on the autonomous control of the hybrid CTR and the design of a lightweight and compact actuator unit in order to ensure the integration on the otologic robot RobOtol (Collin Medical, Bagneux, France).

\section{REFERENCES}

[1] L. Gaillardin, E. Lescanne, S. Morinière, J.-P. Cottier, and A. Robier, "Residual cholesteatoma: prevalence and location. follow-up strategy in adults," European annals of otorhinolaryngology, head and neck diseases, vol. 129, no. 3, pp. 136-140, 2012.

[2] J. Gavin, S. L. Cushing, B. C. Papsin, and A. L. James, "Intraoperative bleeding and the risk of residual cholesteatoma: a multivariate analysis," Otology \& Neurotology, vol. 38, no. 4, pp. 529-534, 2017.

[3] H. Lim, J.-M. Han, J. Hong, B.-J. Yi, S. H. Lee, J. H. Jeong, N. Matsumoto, M. Oka, S. Komune, and M. Hashizume, "Image-guided robotic mastoidectomy using human-robot collaboration control," in 2011 IEEE International Conference on Mechatronics and Automation. IEEE, 2011, pp. 549-554.

[4] B. Dahroug, B. Tamadazte, S. Weber, L. Tavernier, and N. Andreff, "Review on otological robotic systems: Toward microrobotassisted cholesteatoma surgery," IEEE reviews in biomedical engineering, vol. 11, pp. 125-142, 2018.

[5] J. L. Sheehy, D. E. Brackmann, and M. D. Graham, "Cholesteatoma surgery: residual and recurrent disease: a review of 1,024 cases," Annals of Otology, Rhinology \& Laryngology, vol. 86, no. 4, pp. 451-462, 1977.

[6] R. F. Labadie, P. Chodhury, E. Cetinkaya, R. Balachandran, D. S. Haynes, M. R. Fenlon, A. S. Jusczyzck, and J. M. Fitzpatrick, "Minimally invasive, image-guided, facial-recess approach to the middle ear: demonstration of the concept of percutaneous cochlear access in vitro," Otology \& Neurotology, vol. 26, no. 4, pp. 557-562, 2005.

[7] M. Miroir, Y. Nguyen, J. Szewczyk, S. Mazalaigue, E. Ferrary, O. Sterkers, and A. B. Grayeli, "Robotol: from design to evaluation of a robot for middle ear surgery," in 2010 IEEE/RSJ International Conference on Intelligent Robots and Systems. IEEE, 2010, pp. 850-856.

[8] L. Fichera, N. P. Dillon, D. Zhang, I. S. Godage, M. A. Siebold, B. I. Hartley, J. H. Noble, P. T. Russell, R. F. Labadie, and R. J. Webster, "Through the eustachian tube and beyond: A new miniature robotic endoscope to see into the middle ear," IEEE robotics and automation letters, vol. 2, no. 3, pp. 1488-1494, 2017.

[9] T. S. Rau, J. Granna, T. Lenarz, O. Majdani, and J. Burgner-Kahrs, "Tubular manipulators: a new concept for intracochlear positioning of an auditory prosthesis," Current Directions in Biomedical Engineering, vol. 1, no. 1, pp. 515-518, 2015.

[10] J. R. Clark, L. Leon, F. M. Warren, and J. J. Abbott, "Magnetic guidance of cochlear implants: Proof-of-concept and initial feasibility study," Journal of Medical Devices, vol. 6, no. 3, 2012.

[11] S. Weber, K. Gavaghan, W. Wimmer, T. Williamson, N. Gerber, J. Anso, B. Bell, A. Feldmann, C. Rathgeb, M. Matulic et al., "Instrument flight to the inner ear," Science robotics, vol. 2, no. 4, 2017

[12] P. J. Swaney, P. A. York, H. B. Gilbert, J. Burgner-Kahrs, and R. J. Webster, "Design, fabrication, and testing of a needle-sized wrist for surgical instruments," Journal of medical devices, vol. 11, no. 1, 2017.
[13] K. W. Eastwood, P. Francis, H. Azimian, A. Swarup, T. Looi, J. M. Drake, and H. E. Naguib, "Design of a contact-aided compliant notchedtube joint for surgical manipulation in confined workspaces," Journal of Mechanisms and Robotics, vol. 10, no. 1, 2018.

[14] J. Gafford, M. Freeman, L. Fichera, J. Noble, R. Labadie, and R. J. Webster, "Eyes in ears: a miniature steerable digital endoscope for trans-nasal diagnosis of middle ear disease," Annals of Biomedical Engineering, pp. 1-14, 2020.

[15] Y. Chitalia, S. Jeong, N. Deaton, J. J. Chern, and J. P. Desai, "Design and kinematics analysis of a robotic pediatric neuroendoscope tool body," IEEE/ASME Transactions on Mechatronics, vol. 25, no. 2, pp. 985-995, 2020.

[16] C. Girerd, T. Lihoreau, K. Rabenorosoa, B. Tamadazte, M. Benassarou, L. Tavernier, L. Pazart, E. Haffen, N. Andreff, and P. Renaud, "In vivo inspection of the olfactory epithelium: Feasibility of robotized optical biopsy," Annals of Biomedical Engineering, vol. 46, no. 11, pp. 19511961, 2018

[17] J. Granna, T. S. Rau, T.-D. Nguyen, T. Lenarz, O. Majdani, and J. Burgner-Kahrs, "Toward automated cochlear implant insertion using tubular manipulators," in Medical Imaging 2016: Image-Guided Procedures, Robotic Interventions, and Modeling, vol. 9786. International Society for Optics and Photonics, 2016, p. 97861F.

[18] Z. Mitros, S. Sadati, C. Seneci, E. Bloch, K. Leibrandt, M. Khadem, L. da Cruz, and C. Bergeles, "Optic nerve sheath fenestration with a multi-arm continuum robot," IEEE Robotics and Automation Letters, vol. 5, no. 3, pp. 4874-4881, 2020.

[19] C. Girerd, K. Rabenorosoa, and P. Renaud, "Combining tube design and simple kinematic strategy for follow-the-leader deployment of concentric tube robots," in Advances in Robot Kinematics 2016. Springer, 2018, pp. 23-31.

[20] U. Fisch, J. May, T. Linder et al., Tympanoplasty, mastoidectomy, and stapes surgery. Thieme New York, 2008.

[21] P. Amorim, T. Moraes, J. Silva, and H. Pedrini, "Invesalius: an interactive rendering framework for health care support," in International symposium on visual computing. Springer, 2015, pp. 45-54.

[22] C. E. Stepp and S. E. Voss, "Acoustics of the human middle-ear air space," The Journal of the Acoustical Society of America, vol. 118, no. 2, pp. 861-871, 2005 .

[23] S. Y. Ryu, H. Y. Choi, J. Na, E. S. Choi, and B. H. Lee, "Combined system of optical coherence tomography and fluorescence spectroscopy based on double-cladding fiber," Optics letters, vol. 33, no. 20, pp. 23472349, 2008.

[24] J. W. Hamilton, "Efficacy of the ktp laser in the treatment of middle ear cholesteatoma," Otology \& Neurotology, vol. 26, no. 2, pp. 135-139, 2005.

[25] H. B. Gilbert, D. C. Rucker, and R. J. Webster III, "Concentric tube robots: The state of the art and future directions," in Robotics Research. Springer, 2016, pp. 253-269.

[26] T. C. Hudson, M. C. Lin, J. Cohen, S. Gottschalk, and D. Manocha, "V-collide: Accelerated collision detection for vrml," in Proceedings of the second symposium on Virtual reality modeling language, 1997, pp. 117-ff.

[27] C. Bergeles, A. H. Gosline, N. V. Vasilyev, P. J. Codd, J. Pedro, and P. E. Dupont, "Concentric tube robot design and optimization based on task and anatomical constraints," IEEE Transactions on Robotics, vol. 31, no. 1, pp. 67-84, 2015.

[28] D. C. Rucker, R. J. Webster III, G. S. Chirikjian, and N. J. Cowan, "Equilibrium conformations of concentric-tube continuum robots," The International journal of robotics research, vol. 29 , no. 10, pp. 1263$1280,2010$.

[29] EUROFLEX. Nitinol superelastic tubing and stainless steel 316 data sheet. [Online]. Available: https://www.euroflex.de/en/downloads.html

[30] R. J. Webster, A. M. Okamura, and N. J. Cowan, "Toward active cannulas: Miniature snake-like surgical robots," in 2006 IEEE/RSJ international conference on intelligent robots and systems. IEEE, 2006, pp. 2857-2863.

[31] M. Pourafzal, H. A. Talebi, and K. Rabenorosoa, "Piecewise constant strain kinematic model of externally loaded concentric tube robots," Mechatronics, vol. 74, p. 102502, 2021.

[32] E. Arrospide, I. Bikandi, I. García, G. Durana, G. Aldabaldetreku, and J. Zubia, "Mechanical properties of polymer-optical fibres," in Polymer Optical Fibres. Elsevier, 2017, pp. 201-216.

[33] G. Wang, N. M. Le, X. Hu, Y. Cheng, S. L. Jacques, H. Subhash, and R. K. Wang, "Semi-automated registration and segmentation for gingival tissue volume measurement on $3 \mathrm{~d}$ oct images," Biomedical Optics Express, vol. 11, no. 8, pp. 4536-4547, 2020. 